\title{
Carnets
}

Revue électronique d'études françaises de l'APEF

Deuxième série - 11 | 2017

Les écrivains écrivent l'Europe en français

\section{Quand la fiction de Jean-François Dauven flâne en Europe . À propos de Ceux qui marchent dans les villes}

José Domingues de Almeida

\section{OpenEdition}

Journals

Édition électronique

URL : http://journals.openedition.org/carnets/2299

DOI : $10.4000 /$ carnets.2299

ISSN : 1646-7698

Éditeur

APEF

Référence électronique

José Domingues de Almeida, "Quand la fiction de Jean-François Dauven flâne en Europe . À propos de Ceux qui marchent dans les villes », Carnets [En ligne], Deuxième série - 11 | 2017, mis en ligne le 30 novembre 2017, consulté le 02 mai 2019. URL : http://journals.openedition.org/carnets/2299; DOI : $10.4000 /$ carnets.2299

Ce document a été généré automatiquement le 2 mai 2019.

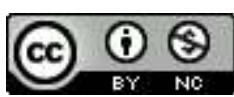

Carnets est mis à disposition selon les termes de la licence Creative Commons - Atribution - Pas d'utilisation commerciale 4.0 International. 


\title{
Quand la fiction de Jean-François Dauven flâne en Europe. À propos de Ceux qui marchent dans les villes ${ }^{1}$
}

\author{
José Domingues de Almeida
}

1 Il convient avant tout d'inscrire Jean-François Dauven dans le contexte littéraire contemporain de la Belgique francophone, marqué par le dépassement des débats identitaires du tournant des années quatre-vingt connus sous le nom de belgitude (Almeida, 2013), mais aussi dans le cadre de la fiction narrative actuelle en français, laquelle se réconcilie avec son référent, notamment spatial (Viart \& Vercier, 2005 : 14).

2 Né à Bruxelles en 1978, Jean-François Dauven, publie deux premiers romans, Le Manuscrit de Portosera-la-rouge (2006) et Le soliste (2007) aux éditions Ramsay (réédition J'ai Lu), en situant ces récits dans une ville méditerranéenne imaginaire, Portosera. Le troisième roman, qui nous occupera dans cette étude, Ceux qui marchent dans les villes, est paru chez Flammarion en 2009.

3 Il est, par ailleurs, impossible de ne pas rapprocher à maints égards le parcours littéraire et fictionnel de Jean-François Dauven de celui de son confrère Grégoire Polet. En effet, tous deux sont Bruxellois, ont le même âge, furent camarades de classe et se signalent par un même style narratif fondé sur l'emploi du présent et la correspondance de personnages choraux, alors que leur fiction révèle un même attrait thématique pour la cartographie fictive et affective des villes européennes.

4 Rappelons aussi - et ce n'est guère un détail pour notre propos - que de Ceux qui marchent dans les villes se dégage une certaine approche et représentation cosmopolite du Vieux Continent en tant que personnage et destin collectif, bien au-delà du simple décor spatiotemporel. À ce titre, Jean-François Dauven s'est fait remarquer par la critique, et était très justement en lice pour le Prix du Livre Européen en 2009 pour une certaine conception et représentation de l'Europe. En effet, ce prix littéraire institué en 2007 par l'association Esprit d'Europe, et présidé jusqu'en 2011 par Jacques Delors, et depuis lors par Pascal Lamy, récompense chaque année un roman et un essai exprimant une vision positive de l'Europe, publiés dans l'un des vingt-huit pays membres de l'Union 
européenne au cours de l'année écoulée, et entend promouvoir les valeurs de l'Europe et contribuer à mieux incarner l'Union auprès des citoyens européens. Il n'est pas insignifiant que Jean-François Dauven ait été choisi pour représenter la Belgique dans l'ouvrage collectif publié sous l'égide de l'Union européenne, intitulé Le dialogue interculturel et le rôle des écrivains dans la promotion de la diversité2.

Cette image positive de l'Europe passe dans le cas de Ceux qui marchent dans les villes (Dauven, 2009) par une grille de lecture éminemment topophilique (Tuan, 1974) des espaces urbains, perçus non pas comme purs décors, mais plutôt comme lieux symboliques, habités par de petites anecdotes enchevêtrées, des destins de flâneurs ; c'està-dire une lecture attentive à l'inscription spatiale du récit dans l'acception géocritique (Westphal, $2007: 15$ ).

6 Par ailleurs, la fiction narrative de Dauven (et de Polet) se signale par des ressemblances génériques référées par la critique au « roman choral », cette structure romanesque où un ensemble de voix se mêlent, s'intègrent ou se cachent, voire se superposent dans une complexe articulation de personnages qui se complètent ${ }^{3}$. D'où la difficulté à résumer cet assemblage narratif.

7 Cela dit, Isabelle Roche s'y est essayée :

À Lisbonne, Jérôme soupire après Marie. Elle, à Paris, ignore où est Jérôme. Elle disparaît et cause bien du souci à Annie, elle-même en train de contempler le désastre de son couple et le lent naufrage de son restaurant. À Rome, Salvatore se sent seul sans Myriam - il essaie bien de rendre heureux leur fils Nicolas, mais il lui faut garder dans ses pensées une place pour les machines à espresso haut de gamme qu'il conçoit et vend avec son associé Giuseppe : ils visent une implantation sur le marché espagnol. À Séville, le concessionnaire des machines La Romana n’a pas la tête à sa recherche de nouveaux locaux : sa femme est sous le charme d'un brillant chef d'orchestre et a refusé de quitter Oviedo pour le suivre. Mais ce jeune chef n'a plus longtemps à travailler là-bas : il est le frère d'une employée de la commission qui vient d'accorder une subvention au théâtre où il est en résidence. Et le directeur, pour conserver le droit aux subsides, doit rompre son contrat. Ladite commission siège à Bruxelles, où Arnaud a cru conquérir Sarah en favorisant son frère - il a bétonné le dossier grâce auquel le théâtre d'Oviedo pourra être subventionné. Et Sarah raconte toute l'affaire par courriel à sa sœur Myriam, partie de Rome pour aller vivre à Prague (Roche, 2009 : 77).

8 La plume narrative de Ceux qui marchent dans les villes se signale par la récurrence du présent de l'indicatif ainsi que par la profusion des personnages, dont un transcendant et prégnant, la ville, ce qui autorise une lecture spatiale et géocritique du récit en tant que lieu habité et vivant (Westphal, 2007 : 15-18) dans la mesure où «l'espace transposé en littérature influe sur la représentation de l'espace dit réel (référentiel) » (Westphal, 2000 : 21), mais surtout de la ville en tant que système sémiotique (Roelens, 2016).

D'autant plus que ce récit est fondé - ne fût-ce que par le titre on ne peut plus explicite sur un exercice de mobilité urbaine. Dans son approche de l'imaginaire poétique urbain, Pierre Sansot rappelait l'importance de la marche dans la ville dans l'appréhension de l'expérience : «La ville se compose et se recompose, à chaque instant, par les pas de ses habitants. Quand ils cessent de la marteler, elle cesse de battre pour devenir machine à dormir, à travailler, à obtenir des profits ou à user son existence » (Sansot, 1971 : 139).

Mais le besoin et le rythme de la marche viennent combler un manque affectif, et mettre les personnages en phase avec la ville, ses rues, ses places, ses fontaines, ses monuments, sa sémiotique interne. Sansot l'exprimait en ces termes: «(...) nous sommes enfermés 
dans une ville à laquelle notre destin est lié dans la mesure où nous avons quelque connivence avec elle» (idem: 138). Et, en effet, plusieurs constantes thématiques et narratives cimentent le roman et cette complicité. Tout d'abord, tous les personnages sont justement marqués par le manque et l'absence, des amours en suspens et des rendezvous manqués. C'est le cas de Jérôme, qui a maladroitement fui Paris et Marie, sa fiancée, à qui il envoie des lettres d'amour et de réconciliation, et que celle-ci ne découvrira qu'à la fin de l'histoire, assurant ainsi la circularité du récit (Dauven, 2009 : 302). Il séjourne à Lisbonne comme guide touristique.

11 Et puis, il y a cet hymne à une Europe à la fois unique et plurielle, mise en fiction à la faveur de l'évocation de dix villes européennes, dont une fictive, Portosera, tout comme est fictif l'écrivain cité en épigraphe, l'introuvable Jièce ; des villes auxquelles l'auteur attribue des épithètes pleines d'authenticité et de vécu : Lisbonne est dite « inoubliable » ; Bruxelles, «méconnue» ; Prague, «énigmatique» ; Séville, «aveuglante» ; Paris, « indicible »; Londres, « inépuisable »; Marseille, « exubérante »; Oviedo, « inattendue », alors que Portosera est forcément sa « favorite»

Portosera, ville imaginée et « imaginaire » (Rondaut, 1990) s'avère, au dire de Dauven, un

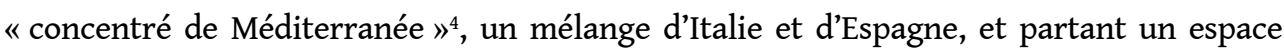
doté d'une certaine référentialité, une ville imaginaire, mais dont le réfèrent est bel et bien réel (idem: 23). Et Portosera gagne dès lors tous les traits fantasmés d'une ville d'Europe du sud. «Cette ville » (Dauven, 2009: 125) est foncièrement aquatique : « Par la fenêtre, Jacqueline contemple la fontaine. Il n'y a que de l'eau dans cette ville, songe-telle : La mer, le fleuve, des fontaines partout (ibidem). En plus, il y a une baie, une île, un vieux port et une cathédrale (idem: 128-131). Nous avons affaire à une véritable projection mentale de la ville idéale et rêvée : « La ville est belle, c'est vrai, c'est peut-être la plus belle du monde (...)» (idem : 140) ; voire utopique par certains aspects : « Quatorze heures. Il fait une chaleur accablante dans toute la ville, première d'Europe à avoir interdit la climatisation pour raisons écologiques » (idem : 144).

Par ailleurs, d'autres passerelles permettent au texte de passer entre ces différentes villes européennes. Il est question d'une machine à café, motif dont la commercialisation fait circuler certains personnages, ou en relie d'autres entre les différentes villes, et que l'auteur affirme beaucoup apprécier en Italie ${ }^{5}$ : «Vous voyez cette machine? Vous voyez comme elle s'intègre au décor? Un modèle unique, conçu tout exprès » (idem : $132 ; c f$. aussi 141, 142, 152, 163, 184, 197, 204, 217, 220).

14 Et puis, il y a aussi cet été torride et caniculaire qui semble unifier et fédérer tout le continent européen, de Lisbonne - «C'est l'été aujourd'hui et la radio annonce une vague de chaleur sur toute l'Europe » (idem : 12) jusqu'à Paris : « (...) malgré le soleil qui inonde Paris » (idem : 272), en passant par toutes les villes évoquées dans leur régime diurne et solaire ( $c f$. idem : 41, 72, 89, 97, 154). Même Londres, connoté au brouillard et à la pluie, «(...) semble toujours étonné par le soleil et la chaleur. Les gens sortent plus tôt des bureaux et rentrent chez eux plus tard, flânent" (idem : 184). L'évolution du soleil ponctue les occupations de la journée, ses rythmes et notamment l'envie irrépressible de sortir en ville, de marcher et de flâner.

En outre, tous les personnages jouissent d'une connaissance authentique des villes dont ils parcourent les rues dans une flânerie qui trahit un attachement passionnel, à Lisbonne comme aux neuf autres lieux européens habités et aimés, et qui se décline selon une cartographie urbaine intime et affective, inscrite dans la fiction. 

la ville : "L'amour de la ville plus que tout autre sentiment se prête à une réduction possible. Il s'agira de montrer qu'il masque autre chose, un désir plus authentique et plus fondamental " (Sansot, 1971: 239). Et Sansot de préciser les prolongements métaphoriques de cet attachement émotionnel à la ville, lesquels gagnent ici en pertinence quand on sait l'état de manque dans lequel se trouvent la plupart des personnages qui se correspondent ou se relaient :

Et, si nous l'admettons, la rencontre de l'être aimé ne précède-t-elle pas aussi celle de la ville? Mais là n'est pas exactement la question. Ne vaut-il pas mieux évoquer un manque d'être, une incomplétude ou un besoin d'être reconnu qui passe parfois par un amour singulier, parfois par une adhésion politique, parfois encore par l'expérience sans réserve d'une ville (idem : 240). externe et épisodique dont les personnages choraux (et Dauven) se méfient systématiquement. Les touristes symbolisent le contraire de la topophilie urbaine, comme le montre leur caricature, et ce indépendamment de la ville. À Lisbonne, «Les Français commentent d'un ton supérieur les arômes du vin portugais » (Dauven, 2009 : 16) ; « Un nouveau groupe est arrivé. Des Français. Vingt-cinq. Une vraie meute. Moyenne d'âge : soixante ans. Les pires» (idem : 36). À Rome, «Cité d'art ? C'est ce que croient les touristes » (idem : 45), ou encore à Marseille, cette réflexion acerbe : « Le tourisme, c'est tout ce que le vingtième siècle aura inventé d'exaltant (...). Le tourisme, c'est la future fortune de Marseille », regrette le personnage António (idem : 116), tout comme à Prague, où les guides touristiques devancent la vraie découverte de la ville et de ses monuments ( idem : 168), ou encore à Paris où « (...) les touristes se font prendre en photo, appuyés au garde-fou, avec en toile de fond un des plus beaux paysages urbains qu'on puisse rêver, de pierre, d'arbres, d'eau, de lumière " (idem: 285), mais un "décor " qu'Annie, le personnage choral de la scénette, semble la seule à être véritablement à même de comprendre et de savourer, c'est-à-dire d'intégrer dans une cartographie personnelle: «Annie se remplit de Paris pour ne plus penser et un instant l'absolue évidence de la vie s'impose » (idem : 285).

18 À ce propos, Bertrand Westphal posait ainsi le socle de l'approche géocritique : «L'enjeu principal de la géocritique n'est pas d'assurer la médiation vers une œuvre désignée. La géocritique permet d'abord de cerner la dimension littéraire des lieux, de dresser une cartographie fictionnelle des espaces humains » (Westphal, $2000: 34$ ). Or les personnages défilant dans les différentes scénettes de Ceux qui marchent dans les villes reproduisent un plan de la ville censé être partagé, reconnu, voire à nouveau parcouru par le lecteur et par son imaginaire. Westphal le rappelle: «Le plan de la ville est une représentation abstraite ; la représentation concrète est délivrée par l'image mentale. L'espace n'existe que parce qu'il est perçu; tout espace, dès lors qu'il est représenté, transite par l'imaginaire » (idem : 35 ).

19 C'est dire que l'authenticité de la ville est à trouver ailleurs, dans la déambulation intime et chercheuse des espaces vivants et des portraits urbains. Ceci suppose la maitrise des cartographies urbaines et leur subtile reproduction spatiale dans le roman à la faveur de la marche en étroite communion avec l'esprit des lieux. À Lisbonne, Jérôme - qui sait ce que la saudade veut dire (Dauven, 2009: 35), lui qui craint d'avoir perdu Marie pour toujours - se dit amoureux de la Baixa, de toutes les ruelles d'Alfama et du Chiado, alors qu'il « (...) n'aime pas Belém. La ville se perd, là-bas, se délaye. Des terrains vagues tentent 
en vain de se faire passer pour des parcs " (idem : 25). À Rome, l'hyperactif Salvatore ne fait qu'un avec «la plus belle ville qu'il ait jamais vue - la sienne» (idem : 53), qu'il connait par cœur et dont il ne se lasse pas de contempler la beauté :

Salvatore s'installe toujours à l'avant du tram, à côté du chauffeur. Il contemple les montagnes au loin, dans la perspective de la Viale Regina Elena, puis les façades lépreuses du quartier San Lorenzo. À l'angle de la Via dei Sabelli monte chaque matin un petit homme rond (...) (idem : 42-43).

De même pour Bruxelles, que Dauvel connaît si bien et qui est exploré dans tous ses axes principaux (idem : 70-71); pour Marseille et ses repères les plus typiques (Noailles, rue de la République, boulevard des Dames, place de la Joliette) (idem : 95), ou encore Prague, parcouru à partir du café Slavia : «Comment peut-elle [Myriam] aimer Prague à ce point et demeurer incapable de s'y retrouver?» (idem : 150); ou pour le plan détaillé du centre de Londres dans la perspective du Belge, Georges, marcheur et flâneur invétéré : « Alors il descend Shaftesbury Avenue jusque Picadilly, d'où il oblique dans Glasshouse street en direction de l'European Bookshop (...). Il y a bien deux heures qu'il marche, à présent » ( idem : 184-185), ou encore pour les plans détaillés des villes d'Oviedo (idem : 209), de Séville (idem : 248-253) ou de Paris (idem : 276-282), et ce toujours à la faveur de la flânerie.

21 Dès lors, le roman choral de Dauven permet de dégager un projet thématique double. D'une part, et tout comme chez Grégoire Polet, il fait apparaître la ville comme personnage central en tant que lieu, être vivant et affectif que l'on assimile, intègre et respire. On est en effet épris d'une ville comme on tombe amoureux de quelqu'un. À ce sujet, Pierre Sansot avait très bien compris combien «En distinguant le passionné d'une ville et l'amateur intelligent, éclairé des villes, nous verrons mieux à quel point la ville peut ne pas constituer un simple décor mais une passion à vivre jusque dans l'amertume et dans la défaite » (Sansot, $1971: 242$ ). Sansot devait aller plus loin dans son chapitre très justement intitulé « Aimer une ville»: «On pensera que nous personnifions la ville. Il ne nous appartient pas de préciser son statut : de sujet, de quasi-sujet, ou d'objet magnifié ? (...) l'homme véritablement concerné par la ville la traite comme une personne » (idem : 245).

22 Aussi le chapitre «Lisbonne, l'inoubliable » commence-t-il par cette déclaration d'amour : «La Rua da Bica de Duarte Belo est la plus belle de Lisbonne, peut-être du monde » (Dauven, 2009 : 11). Rome se signale par une beauté exclusivement perceptible à ceux qui l'aiment et qui sont à même de déchiffrer sa mystérieuse harmonie, comme le traduit la promenade matinale de Salvatore et de son fils, Nicolas :

Sur la Piazza del Quirinale, Salvatore amène son fils au pied de l'étrange protubérance ronde du palais, où s'ouvrent plusieurs bouches de feu. Des histoires de canons, c'est bien, ça l'intéressera. Pourtant le garçon se détourne rapidement, fasciné par la vue, et désigne Saint-Pierre, à l'horizon. «C'est beau, dit-il, on voit loin. » Salvatore n'imaginait même pas que son fils puisse savoir ce qu'est le beau. Il le prend dans ses bras (idem : 58).

Aimer une ville, la ville, en chaque ville, voilà ce qui relie chacun des personnages choraux. Isabelle Roche dira pertinemment à propos de ce roman que : «Villes-chapitres, elles adviennent et prennent corps à travers le regard, les sensations des personnages » (Roche, 2009 : 77). Cet attachement affectif à l'espace urbain intimement vécu requiert la marche comme contrepartie et offrande individuelles. Pierre Sansot voyait dans la marche, dans toutes ses modalités, la condition sine qua non de l'existence urbaine : «Au premier abord, nous constatons un déficit indéniable. Nous avons autour de nous une cité sans les hommes. Or, à la différence de la nature, une ville n'exige-t-elle pas la rumeur obsédante d'êtres qui marchent (...) ?» (Sansot, $1971: 171)$. 

des peuples du Vieux Continent. À cet égard, Bruxelles, la capitale administrative des institutions européennes, y apparaît sous son jour cosmopolite et eurocrate (l'action se déroule au sein de la Commission européenne (idem : 65)). On y apprend également que : «La place du Luxembourg est devenue une sorte de centre de la zwanze internationale » ( idem : 64), alors que, dans la capitale tchèque, Myriam « (...) aime la pluie, comme on peut l'aimer en Europe (...)» (idem : 157) ; que le passage de Georges à Londres évoque certains clichés européens, car ce personnage "(...) ne se sent pas étranger dans la ville. Au contraire. Ce sont des gens comme lui qui font de Londres ce qu'elle est. À Bruxelles, les eurocrates bavardent. À Rome les touristes contemplent. À Paris on bavarde et on contemple. À Londres on décide» (idem : 192); ou qu'à Oviedo, Samuel, en chef d'orchestre, aspire à une fédération européenne par la musique : « Il a du Vivaldi plein la tête. Il attend le jour où, dans une ville d'Espagne, on admettra qu'un Viennois joue de la musique italienne, au lieu de la confiner par principe au répertoire autrichien. Il attend l'Europe » (idem : 214). Cette Europe bureaucratique aussi, mais pourvoyeuse de subsides ( idem : 241).

Mais une Europe qui affiche et respecte les diversités culturelles, et se dessine comme un kaléidoscope idiosyncrasique : « Pour Samuel, l'Europe se divise en deux parties : celle où on boit assis et celle où on boit debout " (idem : 220), tout en partageant un fond historique et civilisationnel commun. Le détail de l'urbanisme pragois contemplé par Myriam dans sa marche fait appel à une Europe antérieure aux divisions géostratégiques et géopolitiques, subtilement magnifiée, caractérisée par un style véritablement transnational faisant corps avec l'esprit de la ville : « Aux façades de béton de l'époque du pacte de Varsovie succèdent les frontons baroques dont la ville est si fière » (idem : 161).

À cet égard, le multilinguisme et le polyglottisme (européens) sont mis en abyme à Séville à la faveur du titre de thèse de doctorat que Virgilio s'apprête à soumettre puisqu'il est question de «L'influence du multilinguisme sur la structure grammaticale française dans les premiers romans d'Arthur-Stanislas Jièce ${ }^{6}$, écrivain dont on apprend qu'il est d'origine "portoséranèse " (idem : 267), c'est-à-dire inexistant, mais aussi par l'universalité de l'expression musicale dont il est question à plusieurs reprises, et qui quelque part célèbre l'Europe. Même pour la ville inventée de Portosera, la question linguistique s'avère symptomatique d'une appartenance européenne cosmopolite, mais non soumise à la rigueur du globish comme lingua franca : «De nouveau Paula adore 
Portosera, ses trois langues [français, italien et espagnol], son soleil, ses beaux hidalgos » ( idem : 143).

En conclusion, une lecture éminemment géocritique, sur un fond européen de Ceux qui marchent dans villes tantôt nettement affiché, tantôt subtilement évoqué, nous semble légitime, d'autant plus que Jean-François Dauven pointe une certaine vision positive, euphorique, voire eurythmique du Vieux Continent, activée par la marche (Sansot, 1971: 243). En effet, nous avons là une Europe où l'on circule librement, où l'on se voit attribuer des soutiens financiers, où l'on parle la langue de l'autre, où l'on tombe amoureux de l'autre, où l'on parle affaires, musique, tourisme, flânerie, où l'on finit par se retrouver et se comprendre. Qui plus est, une Europe que le soleil réchauffe, anime, où il brille d'Ouest en Est, de Lisbonne à Paris, comme un astre auspicieux veillant sur une entité une, mais plurielle et diverse.

\section{BIBLIOGRAPHIE}

ALMeIDA, José Domingues de (2013). De la belgitude à la belgité. Un débat qui fit date. Bruxelles, Bern, Berlin, Frankfurt am Main, New York, Oxford, Wien : P.I.E. Peter Lang.

DAUVEN, Jean-François (2006). Le manuscrit de Portosera-la-rouge. Paris : Éditions Ramsay.

DAUVEN, Jean-François (2007). Le soliste. Paris : Éditions Ramsay.

DAUVEN, Jean-François (2009). Ceux qui marchent dans les villes. Paris : Flammarion.

ROCHE, Isabelle (2009). « Petite couronne européenne », Le Carnet et les Instants, nº 157, p. 77.

ROELENS, Nathalie (2016). Lire, écrire, pratiquer la ville. Paris : Kimé.

ROUDAUT, Jean (1990). Les villes imaginaires dans la littérature française. Paris : Hatier.

SANSOT, Pierre (1971). Poétique de la ville. Paris : Klincksieck.

TUAN, Yi-Fu (1974). Topophilia : a study of environmental perception, attitudes, and values. Prentice-

Hall: Englewood Cliffs, NJ.

VIART, Dominique \& VERCIER, Bruno (2005). La littérature française au présent. Héritage, modernité, mutations. Paris : Bordas.

WESTPHAL, Bertrand (2000). La géocritique mode d'emploi. Limoges : PULIM.

WESTPHAL, Bertrand (2007). La géocritique. Réel, fiction, espace. Paris : Minuit.

Sitographie :

http://www.ina.fr/video/3857755001 [disponible le 04/07/2017]

http://ulysseias.ilcml.com/pt/termos/dauven-jean-francois/ [disponible le 04/07/2017]

http://eeas.europa.eu/archives/delegations/algeria/documents/

ce_livre_recontre_ecrivains_fr.pdf [disponible le 04/07/2017] 


\section{NOTES}

1. Cet article s'insère dans la recherche menée au sein du Programme Stratégique intégré UID/ ELT/00500/2013 | POCI-01-0145-FEDER-007339.

2.

http://eeas.europa.eu/archives/delegations/algeria/documents/

ce_livre_recontre_ecrivains_fr.pdf

3. Cf. l'entrée Dauven, J.-Fr. de la base de données Ulyssei@s de l'ILCML http:// ulysseias.ilcml.com/pt/termos/dauven-jean-francois/

4. http://www.ina.fr/video/3857755001

5. ibidem.

6. Virgilio garde d'ailleurs en tête le projet d'un article scientifique sur un sujet qui lui tient à cœur : "Comment un écrivain polyglotte et vivant dans une ville polyglotte n'aurait-il pas une vision du monde plus ouverte? ? (Dauven, 2009 : 268).

\section{RÉSUMÉS}

Nous entendons mettre en exergue la fictionnalisation de l'Europe dans le roman Ceux qui marchent dans les villes (2009) de l'écrivain belge francophone contemporain Jean-François Dauven, à partir de l'enchevêtrement narratif suscité par la flânerie de plusieurs personnages aux destins liés dans Lisbonne, Rome, Bruxelles, Marseille, Prague, Londres, Oviedo, Séville, Paris, ainsi que dans une ville imaginaire, Portosera; autant de points d'ancrage, mais surtout de flânerie pour dix histoires chorales.

We intend to highlight the fictionalization of Europe in the novel Ceux qui marchent dans les villes (2009) by the contemporary Belgian Francophone writer Jean-François Dauven, This novel is based on the narrative entanglement aroused by the strolling of several characters. Their destinies meet in Lisbon, Rome, Brussels, Marseille, Prague, London, Oviedo, Seville, Paris, as well as in an imaginary city, Portosera, as many anchors, but more specifically spaces of flânerie for ten choral stories.

\section{INDEX}

Mots-clés : Europe, roman choral, flânerie, Dauven, villes

Keywords : Europe, choral novel, flânerie, Dauven, cities

\section{AUTEUR}

\section{JOSÉ DOMINGUES DE ALMEIDA}

Un. Porto - ILCML - APEF

jalmeida@letras.up.pt 